\title{
The Challenge of Providing Evidence-based Transitional Care*
}

Mary D Naylor ${ }^{\mathrm{a}}$

University of Pennsylvania, Estados Unidos

naylor@nursing.upenn.edu

ORCID: https://orcid.org/0000-0001-9287-153X

Mark Toles

University of North Carolina at Chapel Hill, Estados

Unidos

DOI: https://doi.org/10.11144/Javeriana.ie23.cpet

Transitions in care are events that occur as individuals transfer between settings and providers of healthcare. The movement from a hospital to home-based care is a common example of a care transition. Due to common breakdowns between health and social care systems, continuing risks for acute health events, and the lack of support to manage complex treatments at home, between 15-20\% of hospitalized adults are rehospitalized within thirty days of returning home. This high rate of hospital readmissions points up the need for transitional care services to address challenges in continuing care at home.

Transitional care is a set of time-limited set of services designed to ensure care continuity, to avoid preventable poor outcomes among at-risk populations, and to promote the safe and timely transfer of patients from one level of care or from one type of setting to another. The core components of transitional care are:(1) engaging patients and family caregivers (family members, friends, andneighbors), (2) educating patients and their caregivers, (3) managing complex health and social needs, (4) promoting well-being; and (5) assuring continuity and accountability for care. Accumulated evidence demonstrates that high quality transitional services reduce the rate of acute medical events after patients return home. An exemplar of such care is the Transitional Care Model, in which advanced practice nurses engage patients, family caregivers and other team members, teach and support patients and family members at hospital and at home.

Although the efficacy of transitional care is now well-known, our experiences in research and quality improvement suggest three overlapping challenges to assure that patients receive effective transitional care services: (1) the COVID-19 pandemic; (2) health disparities; and (3) the implementation of evidence-based models of care. Indeed, the global public health crisis has exposed longstanding health inequities that have contributed to avoidable poor health outcomes among marginalized individuals and communities and placed a spotlight on the critical need for transitional care solutions grounded in science

COVID-19 created a historic demand for high quality transitional care services. In the U.S., for example, more than 32 million individuals were infected, 2,127,000 were hospitalized, and 580,000 individuals died (as of May 2021). A less known statistic is that nine out of ten adults, who were hospitalized with COVID-19, returned home after. Survivors of COVID-19 are usually older adults with multiple chronic conditions, often complicated by functional deficits. A disproportionate number of those affected by this virus are members of minority groups many of whom also are socially disadvantaged. Moreover, as patients with COVID-19 are isolated in the hospital, COVID survivors and their family caregivers have fewer opportunities to interact with health care clinicians and to develop plans for care at home. Nurses and other clinicians who provide transitional care also experience serious challenges locating community-based partners, such as home care nurses and home care aides. Finally, a major challenge is the enormous burden born by family caregivers, who

Author notes

\footnotetext{
${ }^{a}$ Corresponding author. E-mail: naylor@nursing.upenn.edu
} 
provide critical support throughout transitions in care. While these COVID-related challenges continue, transitional care remains a fundamental service and support for COVID survivors and their families. Rapid advances in the use of telehealth, public health messaging, and infection monitoring have been key resources to meet transitional care needs during the COVID-19 pandemic.

Fundamental challenges to provide transitional care include disparities in access to high quality health care among groups with social, economic and/or environmental disadvantages. These inequities have enormous negative human and economic consequences. For example, findings from a large U.S. cohort study revealed that the rehospitalization rate of older adults from the most disadvantaged US neighborhoods were significantly higher than national norms, and similar to the rehospitalization rate of individuals with chronic pulmonary disease. Health care disparities create daily challenges for transitional care clinicians who must (1) plan care with individuals who are uninsured or under-insured, (2) teach strategies for managing illness with individuals who cannot afford safe housing and healthy food, and (3) coordinate care in environments where health access and community support is constrained. In the U.S., new approaches to reducing health disparities include increasing access to health insurance coverage and federal initiatives to eliminate racial, ethnic and socioeconomic health inequities. Key advances in providing transitional care have been programs to identify at risk patients, promote their access to health insurance and health and community-based services, and increase the diversity of the workforce and the cultural knowledge of health care clinicians.

An additional challenge to provide transitional care is the continuous need to adjust to changes in the health care system and to adapt transitional care services based on emerging needs and technologies. For example, evidence-based transitional care models, such as the Transitional Care Model, include hospital and in-home services provided by the same, well-trained clinician, such as an advanced practice registered nurse. In practice, however, evidence-based transitional care models often are adapted; thus, transitional care frequently does not include patient contact after hospital discharge, or home visits are replaced with telephone calls. Evidence from rigorous, large-scale studies are needed to demonstrate the effectiveness of these adaptations of transitional care. Additionally, the effectiveness of transitional care is likely commensurate to the implementation of other patient-centered health care services, such as advance care planning as well as support for family caregivers, many of whom currently need to leave the workforce to support seriously ill family members at home. Thus, transitional care must evolve in response to changing societal needs, with greater emphasis on palliative care services and coordination with specialists to manage progressive illnesses, such as dementia. Finally, unique adaptations of transitional care must be developed in community-based settings, such as skilled nursing facilities and home care with emerging technology, such as remote patient monitoring and telehealth systems.

Since the early 1990's, patients experiencing transitions in care and clinicians and researchers have continuously collaborated, by creating paths for the continuity of care that people need to improve their health and quality of life. While transitional care science continuesevolving, available evidence reveals many opportunities for immediate improvement. What is needed now is our resolve and will to bridge gaps in health and social services and provide patients with equitable access to the high quality, evidence-based transitional care that aligns with their goals and supports their family caregivers.

\section{Notes}

* $\quad$ Editorial

Licencia Creative Commons CC BY 4.0

How to cite this editorial: Naylor MD, Toles M. The challenge of providing evidence-based transitional care. Investig Enferm Imagen Desarr. 2021;23. https://doi.org/10.11144/Javeriana.ie23.cpet 